\title{
Article
}

\section{Extensional Flow of Carbon Nanotube Dispersion and Its Influence on Electrical Conductivity}

\author{
Nono Darsono, Hiroshi Mizunuma ${ }^{\dagger}$, and Hiromichi OBara \\ Department of Mechanical Engineering, Tokyo Metropolitan University, \\ 1-1 Minamiohsawa Hachioji, Tokyo, Japan 192-0397 \\ (Received : December 7, 2011)
}

\begin{abstract}
We herein describe our investigation of the rheological and electrical characteristics of single- and multi-walled carbon nanotube dispersions. The effect of the viscosity of the dispersing liquids was investigated under shear. At low shear rates, a dispersing liquid with a low viscosity caused the relative viscosity to increase more than a dispersing liquid with a high viscosity, in the case of single-walled carbon nanotube dispersions. The shear viscosity approached the same value regardless of the viscosity of the dispersing liquid. The independence of the shear viscosity suggests that at low shear rates it is dominated by the microstructure of the single-walled carbon nanotubes rather than the viscosity of the dispersing liquid. Extensional flow was produced by the capillary thinning of a liquid thread, and both the apparent extensional viscosity and the electrical conductivity were measured for the carbon nanotube dispersions. During capillary thinning, the apparent extensional viscosity approached the theoretical extensional viscosity predicted by Batchelor for low concentrations, but was notably higher than the theoretical values predicted for high concentrations. The electrical conductivity was increased via the stretching of the threads and the solidification of the dispersing photopolymer. The concentration at the electrical percolation threshold was approximately the same as the concentration at which the apparent extensional viscosity deviated from the theoretical prediction of Batchelor. A network structure of carbon nanotubes is herein suggested to increase the apparent extensional viscosity at concentrations above the percolation threshold.
\end{abstract}

Key Words: Stretching / Extensional viscosity / Carbon nanotubes / Conductivity / Percolation threshold / Photopolymer

\section{INTRODUCTION}

Carbon nanotubes (CNTs) have been studied extensively ever since their discovery by Iijima ${ }^{1)}$, because of their potential use in various applications, especially in electronics and polymer composites. The mechanical and electrical characteristics of CNT polymer composites have been summarized in a number of review papers. ${ }^{2-6)}$ These characteristics generally reflect the nanostructure, large surface area, and high electrical conductivity of CNTs. The aspect ratios of CNTs are generally of the order of $10^{2}$ to $10^{3}$, and their alignment therefore influences the electrical and mechanical characteristics of the sample in question. For example, well aligned CNTs are used as efficient field emitters $^{7,8)}$ and these are therefore incorporated in some applications such as display screens. The screen printing of CNT pastes is a low cost technique used in the fabrication of field emitters, but a technique such as napping using an adhesive tape $\mathrm{e}^{9)}$ is additionally required to expose the CNTs

$\dagger$ mizunuma@tmu.ac.jp perpendicular to the surface. The alignment of CNTs in a polystyrene matrix results in higher yield and ultimate strengths compared to randomly oriented composites, as reported by Thostenson and Chou. ${ }^{10)}$ Therefore, the control of the alignment of CNTs is important if satisfactory mechanical and electrical properties are to be achieved.

A well known means of aligning rod-like particles or polymer chains along a single direction is via extensional flow, and this, or composite stretching, is an effective method of controlling the alignment of CNTs. Wang et al. ${ }^{11)}$ stretched CNT composites and discussed their anisotropic mechanical and electrical properties. Rheological studies of extensional flow were undertaken by Ma et al. ${ }^{12)}$ and Tiwari et $a l .{ }^{13)}$, who discussed a rheological model of CNT dispersions under capillary thinning of a liquid thread. This rheometry of capillary thinning is a method that has been widely used to measure extensional viscosity, as reviewed by Mckinley. ${ }^{14)}$ The stretching and capillary thinning of CNT dispersions are suitable means of controlling and analyzing the alignment of CNTs.

Some use has previously been made of percolation theory to analyze particle contact in dispersed systems, whose electrical 
properties are closely related to the characteristics of the contacts between CNTs. Percolation theory has therefore been used to study the electrical properties of CNT composites, as reviewed by Bauhofer and Kovacs. ${ }^{4)}$ The so-called 'percolation threshold' corresponds to the CNT concentration at which the contact paths of the CNTs are complete throughout the dispersed system. CNT composites have very low percolation thresholds compared with other fillers, such as graphite or carbon fiber. At the same time, the percolation threshold is also influenced by factors such as CNT type and dispersion method, as well as the composite polymer used. However, percolation theory is a relatively sophisticated means of analyzing CNT dispersion. By contrast, rheological studies offer an alternative important means of analyzing and controlling CNT dispersions. In our previous study ${ }^{15}$, shear flow rheometry was applied to characterize the photosolidification of CNT dispersions. Rheological studies can also play an important role in improving the mechanical and electrical properties of CNT dispersions.

In our present study, we investigated the rheological and electrical characteristics of a CNT-dispersed photopolymer. Under shear flow, we measured the effect of using different viscosities of dispersing photopolymers. Extensional flow conditions were produced by the capillary thinning of a liquid thread, which allowed us to measure both the apparent extensional viscosity and the electrical conductivity. The alignment of the CNTs in the dispersion was fixed by the irradiation of UV light and observed using an electron microscope. We herein discuss the percolative characteristics of the dispersions in terms of their observed rheological and electrical properties.

The remainder of the paper is organized as follows. In Section 2, we describe the experimental method. In Section 3 , we discuss the results, and we present our conclusions in Section 4.

\section{EXPERIMENTAL METHOD}

\subsection{Material}

The monomer of the photopolymer used was alkoxylated neopentyl glycol diacrylate (CD9043, Sartomer Company Inc.), and a photoinitiator (Irgacure 819, BASF Japan Ltd.) was added to the monomer at a concentration of $5 \mathrm{wt} \%$. The viscosity of this combination was $0.071 \mathrm{~Pa} \cdot \mathrm{s}$. Apart from CD9043, two other photopolymers were also used as dispersing liquids for CNTs. The photopolymer UAP-75 (Afit Corp.) is mainly composed of 2.2 bis 4-(acryloxy diethoxy) phenyl, tricylodecane dimethanol diacrylate monomer, and here we mixed it with a photoinitiator (a mixture of Irgacure 184 and 819, BASF Japan Ltd.) at a concentration of $1 \%$, together with a thickener. The resulting viscosity of the UAP75 was $3.12 \mathrm{~Pa} \cdot \mathrm{s}$. The third photopolymer used was IB-XA (Kyoeisha Chemical Co. Ltd.), which is formed from the monomer isobornyl acrylate, together with a photoinitiator (Irgacure 819, BASF Japan Ltd.) at a concentration of $5 \mathrm{wt} \%$, and its resulting viscosity was $0.011 \mathrm{~Pa} \cdot \mathrm{s}$. The viscosity of the photopolymer UAP-75 was higher than that of CD9043, and the viscosity of IB-XA was lower than that of CD9043, and in neither case could their extensional viscosity be measured because capillary thinning was incomplete. For this reason, the CNT dispersions of UAP-75 and IB-XA were only tested by measuring their shear viscosities.

The dispersed CNTs consisted of single-walled carbon nanotubes (SWCNTs, Nikkiso Co. Ltd.) and multi-walled carbon nanotubes (MWCNTs, VGCF-X, Showa Denko K. K.). The diameter and length of the SWCNTs were typically $2 \mathrm{~nm}$ and a few microns, respectively. Figure 1(a) shows an electron micrograph of the spaghetti-like SWCNTs. The diameter and length of the MWCNTs were typically $15 \mathrm{~nm}$ and $3 \mu \mathrm{m}$, respectively. These CNTs were all produced using a chemical vapor deposition (CVD) process. The SWCNTs were supplied in the form of buckypaper, and the MWCNTs were supplied as a fine powder. The MWCNTs may be seen as curled shapes in Fig 1(b).

The CNTs were firstly dispersed in acetone, and an ultrasonicator (Branson Sonifier 150) was then applied for a total of 12 hours in order to achieve uniform dispersion. The dispersion was maintained between 0 and $4{ }^{\circ} \mathrm{C}$ in a temperature-controlled bath, and the evaporation of acetone was prevented during ultrasonication. The dispersed CNTs were then dried in a convection oven at $70{ }^{\circ} \mathrm{C}$, after which they were mixed with photopolymer for one hour.

\subsection{Measurements of Rheological and Electrical Characteristics}

The steady shear viscosities were measured using a cone and plate rheometer (RS 600, Thermo Haake). The diameter of the cone was $20 \mathrm{~mm}$ and the gap angle was $1^{\circ}$. The extensional rheological properties were measured in terms of the capillary thinning of a liquid thread. A liquid bridge was set up between parallel disks $1.5 \mathrm{~mm}$ in diameter, as shown in Fig. 2. The resulting liquid thread was monitored using a CCD video camera (BU-40C, BITRAN Corp.), and the initial diameter $D_{0}$ of the liquid bridge was adjusted to between 1.2 and $1.5 \mathrm{~mm}$. The initial distance between the disks $L_{\mathrm{o}}$, and therefore the length of the liquid bridge, was $0.46 \mathrm{~mm}$. The 
disks were connected to two linear motors that stretched the liquid between the disks. The midpoint of the liquid thread was maintained at the same height during stretching. The disks were brought to a stop at a disk separation $L$ of $1.46 \mathrm{~mm}$, yielding a value of $L / L_{0}$ of 3.2. The aspect ratio $\Lambda_{0}\left(=L_{0} / D_{0}\right)$ of the initial liquid bridge must be lower than the Rayleigh's statistically-stable ratio $\pi$. In this study, values of $\Lambda_{0}(=0.31$ to 0.38 ) and $L / L_{0}$ were determined from preliminary tests and in consideration of previous studies ${ }^{16-18)}$ in which the ranges of $\Lambda_{0}$ and $L / L_{0}$ were $0.5-1$ and $2.3-3.7$, respectively.

In order to apply a constant extensional rate $\dot{\varepsilon}$ of $74 \mathrm{~s}^{-1}$, we determined the separation velocity $V$ of the two disks using

$$
V=\dot{\varepsilon} L_{0} e^{\dot{\varepsilon} t}
$$

where $t$ is the time after the start of the motor. After stopping the motor, the liquid thread was thinned by capillary action, and the transient variations in thread diameter were measured using an LED/CCD optical micrometer (LS-7000, Keyence Inc.). The resolution of these measurements was $3 \mu \mathrm{m}$ and the transient variations in diameter at the midpoint were recorded
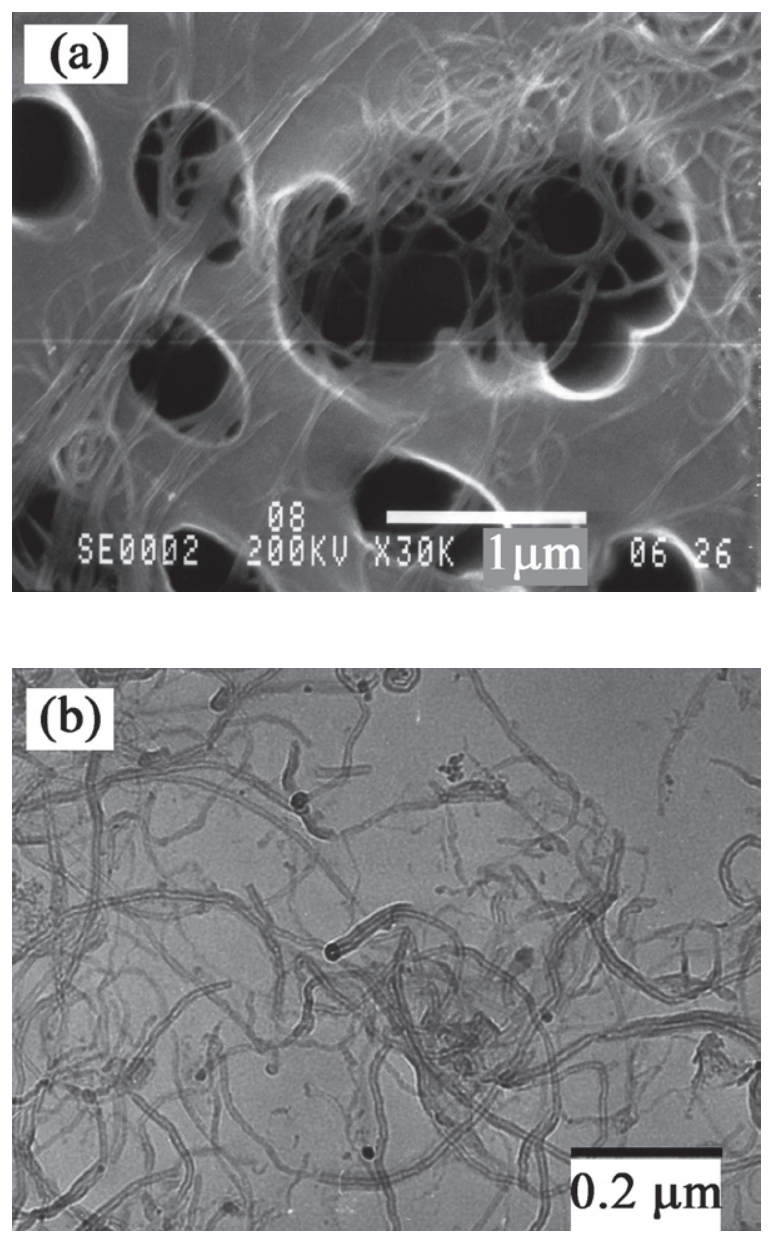

Fig. 1. Electron micrographs of the CNTs showing (a) SWCNTs and (b) MWCNTs. at a rate of $2.5 \mathrm{~ms} / \mathrm{sample}$.

An apparent extensional viscosity $\eta_{E}$ may be obtained from the rate of change in the mid-diameter $D_{\text {mid }}$ induced by viscous capillary thinning. In a Newtonian fluid, $\eta_{E}$ is given by

$$
\eta_{E}(t)=(2 X-1) \frac{\sigma}{\left(-\frac{d D_{\text {mid }}(t)}{d t}\right)}
$$

where $\sigma$ is the surface tension and $X$ is a constant. McKinley and Tripathi ${ }^{16)}$ discussed capillary thinning flow using the results of numerical simulation and experimental observation. They revealed that the final stage prior to the breakup of the filaments was characterized by Eq. (2) with $X=0.7127$. This value of $X$ was originally obtained by Papageorgiou ${ }^{17)}$, who determined this value numerically from the self-similar solution of the inertialess Stokes equations. In our study, we used Eq. (2) to estimate the apparent extensional viscosity $\eta_{E}$ for the SWCNT and MWCNT dispersions. The mid-diameter $D_{\text {mid }}$ decreased linearly (as shown in Fig. 7), and the slope $d D_{\text {mid }}(t) / d t$ in Eq. (2) was therefore assumed to be constant, and was obtained from the plot.

The rheometry of this type of capillary thinning was reviewed in detail by McKinley. ${ }^{14)}$ In this rheometry, the extensional viscosity and the surface tension both play important roles. In addition, it is necessary to minimize the influence of gravity and of the inertial force. The ratio of gravity to the surface tension force is expressed by the Bond number $B o\left(=\rho g R_{0}{ }^{2} / \sigma\right)$, where $\rho$ is fluid density, $\mathrm{g}$ is gravitational acceleration, $R_{0}$ is the initial thread radius, and $\sigma$ is surface tension. A high value of Bo induces gravitational sagging, and should therefore be less than 1 . In this study, $B_{\text {o }}$ was less than 0.18 , and of the same order as the value of 0.0 - 0.6 used in other studies. ${ }^{16,18,20)}$ The effect of viscosity on capillary thinning is dominated by the Ohnesorge number

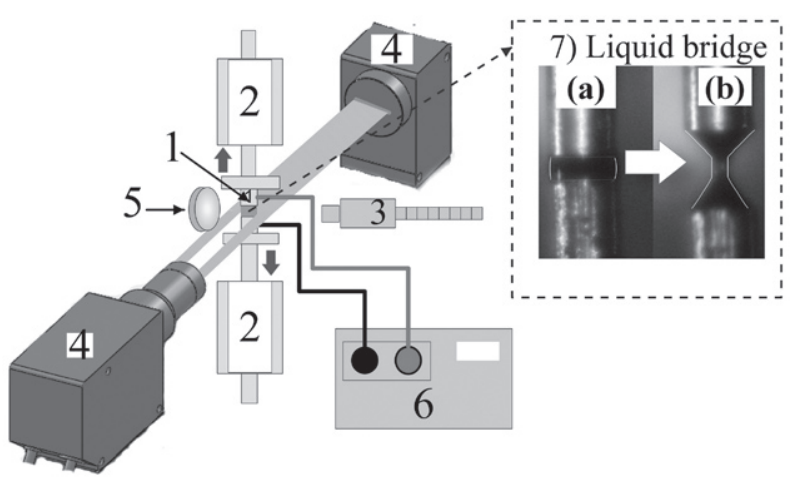

Fig. 2. Setup of the stretching experiment. 1. Stainless steel parallel disk; 2. Linear motor; 3. UV light source; 4. LED/CCD-type digital micrometer; 5 . Concave mirror; 6. Digital electrometer; 7. Liquid bridge (a) before and (b) after stretching. 
$O h\left(=\eta / \sqrt{\rho \sigma R_{0}}\right)$, where $\eta$ is the viscosity. If $O h$ is too low, inviscid capillary thinning occurs, rather than viscous capillary thinning. Rodd et $a l^{21)}$ suggested that for a Newtonian fluid Oh should be greater than 0.14 if viscous capillary thinning is to be achieved. In this study, $O h$ is greater than 0.46 and is of the same order of magnitude as the value of $0.08-0.9$ used in other studies. $^{20,21)}$

We measured the electrical conductivity of the CNTdispersed system during the process of capillary thinning described above. The variation in the electrical resistance $R$ was measured for the thinning thread between the parallel stainless steel electrode disks using a digital electrometer (Model 2100, Keithley Instruments Ltd.), as shown in Fig. 2. This electrometer applied the constant current of $500 \mathrm{nA}$ to material and the electrical resistance outputted was given from the detected voltage. The measurements were carried out on the capillary thinning thread solidified by UV light, and example of which is shown in Fig. 3. The photopolymer was exposed to UV light (LS-165UV, Sumita Optical glass Inc.) at the highest possible intensity to ensure rapid solidification. The controller of the UV light was connected to the diameter sensor and set to start UV irradiation prior to the breakup of the thread. The change in the conductivity was influenced not only by the extension of the thread but also by its solidification. The conductivity of the liquid thinning thread was therefore also measured in the absence of UV light; from these measurements, the influences of both the extension and the solidification could be investigated individually.

The surface tension of the CNT dispersions was measured using a Wilhelmy plate method (automatic tensiometer CBVP-Z, Kyowa Interface Science Co. Ltd.). The surface tension of the CD9043 was $31.2 \mathrm{mN} / \mathrm{m}$, and similar values

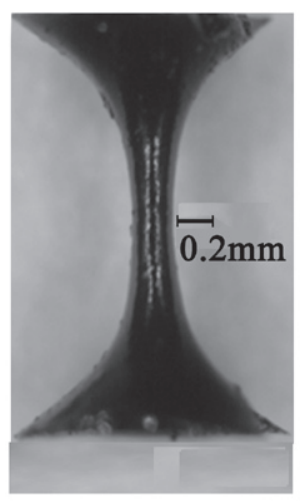

(a)

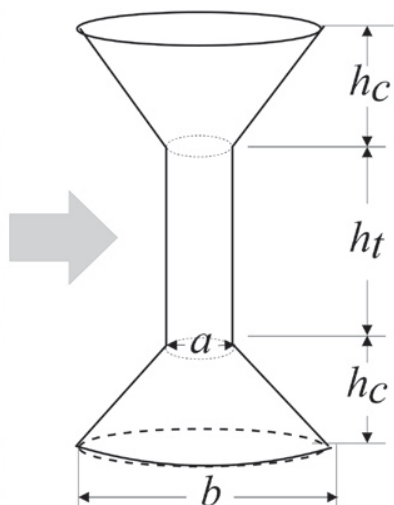

(b)
Fig. 3. Stretched and solidified sample showing (a) sample of photopolymer dispersed CNTs and (b) geometrical definition of the central thread and the truncated cones. were obtained for the MWCNT dispersions. The values ranged from $-1.2 \%$ to $+6.5 \%$, and the surface tension of the dispersed MWCNT was not significantly different to that of the CD9043. The surface tension of the SWCNT dispersion of $0.05 \mathrm{wt} \%$ was $32.1 \mathrm{mN} / \mathrm{m}$; we could not measure the surface tension reliably at higher concentrations because the viscosities were too high. We therefore assumed a surface tension of $32.1 \mathrm{mN} / \mathrm{m}$ for all the SWCNT dispersions.

\section{RESULTS AND DISCUSSION}

\subsection{Rheological Characteristics Under Steady Shear}

Figure 4 shows the variation in steady-state shear viscosity with shear rate for the CNT dispersions of CD9043. The photopolymer without CNTs behaves like a Newtonian fluid, and the dispersed systems show an increase in shear thinning with concentration. As shown in Fig. 4(a), Newtonian plateaus appear at shear rates lower than $1 \mathrm{~s}^{-1}$ and higher than $10^{2} \mathrm{~s}^{-1}$ for the dispersions of SWCNTs. The shear thinning of the dispersions of MWCNTs are shown in Fig. 4(b). In contrast with the results for the SWCNTs, there is no clear Newtonian plateau at low shear rates. The Newtonian plateau
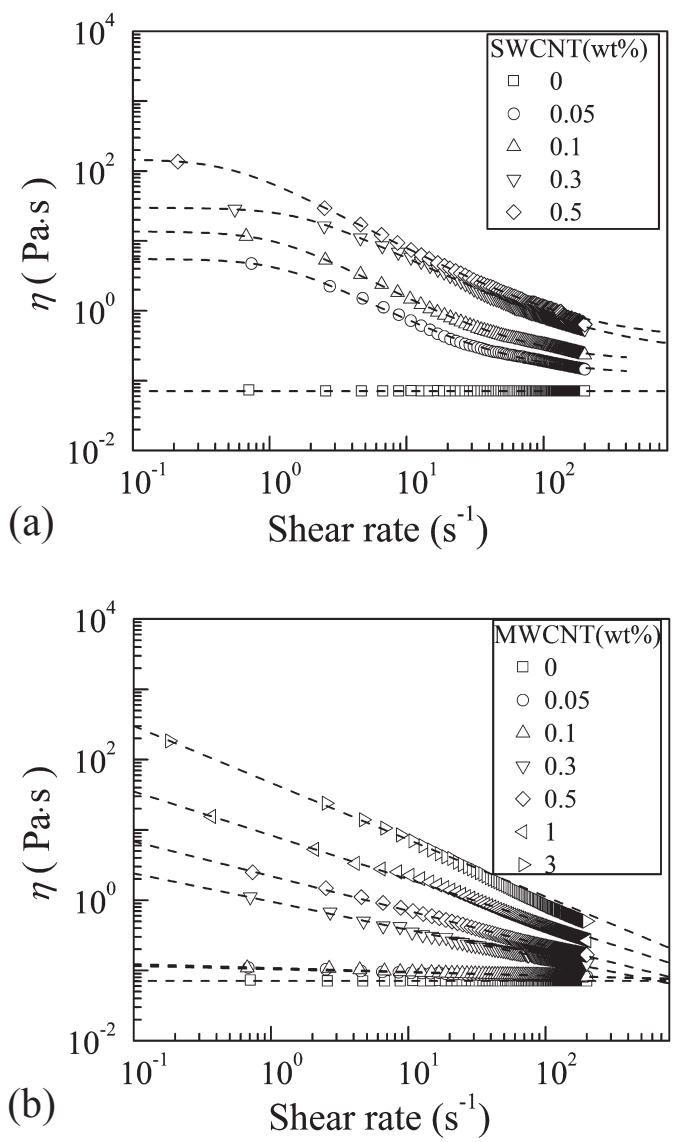

Fig. 4. Steady shear viscosity as a function of shear rate showing (a) SWCNT dispersions and (b) MWCNT dispersions. The dispersing liquid is CD9043. 
for MWCNTs would appear in the lower or higher shear rates than the measured shear rates. The increase in viscosity is less than that seen for the SWCNT dispersions. The presence of SWCNTs brings about viscosities that are thirty times higher than those brought about by the presence of MWCNTs for dispersions of $0.5 \mathrm{wt} \%$.

The relative viscosities $\eta / \eta_{0}$ for the dispersing systems IBXA, CD9043 and UAP-75 are shown in Fig. 5, where the viscosities $\eta_{0}$ of the photopolymers are 0.011 for IB-XA, 0.071 for CD9043, and 3.12 Pa.s for UAP-75. Generally, for spherical particles dispersion increases the relative viscosity as a function of the concentration, and this increase is independent of the viscosity of dispersing liquid. However, for SWCNT systems, the relative viscosity depends on the dispersing photopolymer, and a photopolymer that has a lower viscosity produces a higher relative viscosity. The viscosity of IB-XA is $1 / 280$ of that of UAP-75, and an SWCNT dispersion of $0.5 \mathrm{wt} \%$ has a relative viscosity that is 170 times higher for IB-XA than for UAP-75 at a shear rate of $1 \mathrm{~s}^{-1}$. In contrast, the MWCNT systems showed no clear dependence of relative viscosity on the viscosity of dispersing photopolymer.

As well as relative viscosity, we also show the absolute

(a)

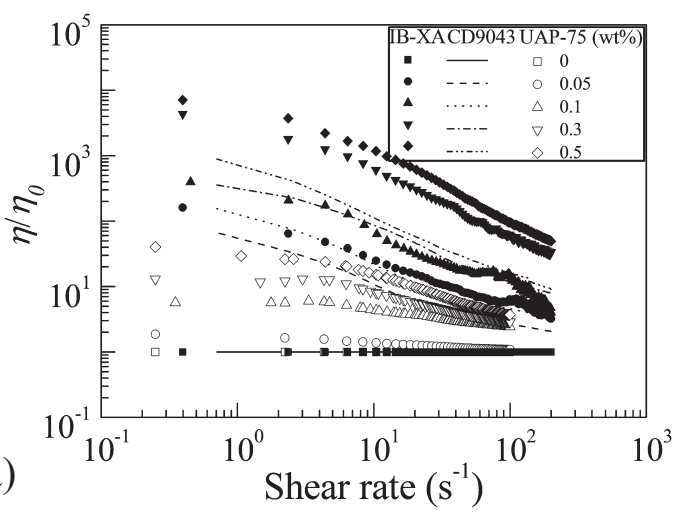

(b)

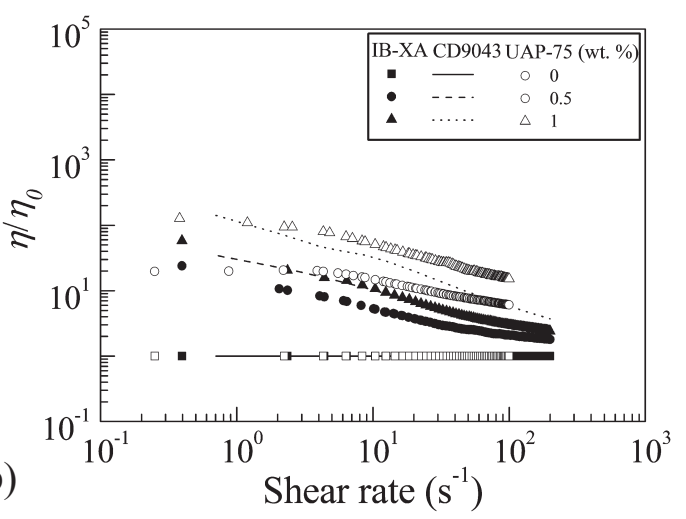

Fig. 5. Relative viscosity as a function of shear rate for (a) SWCNT dispersions and (b) MWCNT dispersions. The dispersing liquids are IB-XA, CD9043, and UAP-75. viscosity as a function of shear stress in Fig. 6. At high shear stress, the viscosity of the dispersion depended on the viscosity of the photopolymer and decreased with the viscosity of the photopolymer. At low shear stress, the range of measurement of shear stress varied depending on the photopolymer used, and it was difficult to compare precisely the viscosity of each dispersion system. However, at low shear stress, the viscosity of each concentration approximately approached the same level regardless of the dispersing photopolymer used. This independence suggests that the shear viscosity was dominated by the microstructure of the CNTs rather than the viscosity of the dispersing liquid. Kinloch et $a l^{22)}$ measured the steady and oscillatory shear viscosity of a water dispersion of MWCNTs, and observed notable increases in $G^{\prime}$ and $G^{\prime \prime}$ after a steady shear rate sweep from 1 to $1000 \mathrm{~s}^{-1}$. They suggested that MWCNTs formed microstructures like flocculated networks. They thought that those microstructures were destroyed by pre-shear and were recovered during linear viscoelastic measurement. The direct observation of the CNT microstructure is somewhat difficult, but an alternative indirect method could be via the investigation of the influence of the viscosity of the dispersing liquid on the rheological
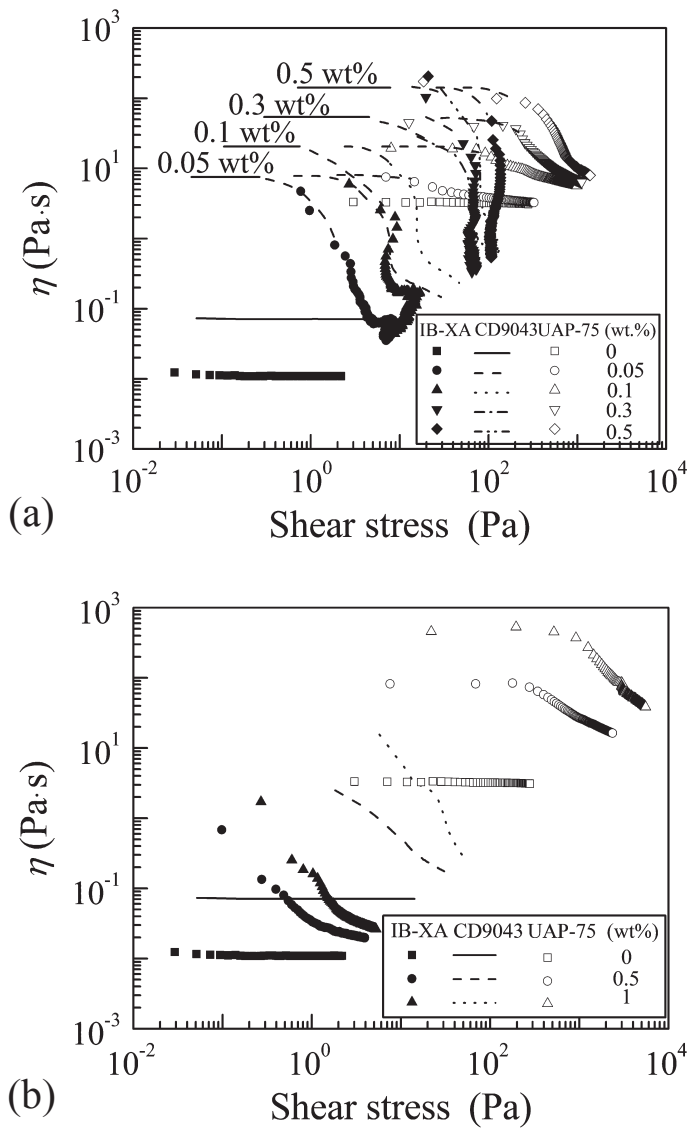

Fig. 6. Viscosity as a function of shear stress for (a) SWCNT dispersions and (b) MWCNT dispersions. The dispersing liquids are IB-XA, CD9043, and UAP-75. 
properties of the dispersions.

\subsection{Rheological Characteristics in Extensional Flow}

In order to investigate the extensional flow of the CNT dispersion, we plotted the variation in the mid-diameter $D_{\text {mid }}$ of the thread (Fig. 7). The action of the linear motors stretched the thread of the dispersion and induced a steep reduction in $D_{\text {mid }}$. After the motors were stopped, capillary thinning began and was clearly influenced by the concentration of CNT. Similar to the variation in shear viscosity, the SWCNT dispersion shows a higher resistance to thread thinning than the MWCNT dispersion for the same concentration. The thinning thread was solidified by UV light, and the cross section of the solidified thread was observed using an electron microscope; a typical example is shown in Figure 8. The cross section was made in the direction perpendicular to the axis of stretching. The CNTs may be seen to protrude from the cross section. The appearance of the protruded CNTs is similar to the micrograph in Fig. 1, in that whereas the SWCNTs are almost straight, the MWCNTs are rounded in shape.

Figure 9 shows the apparent extensional viscosity $\eta_{E}$ as a
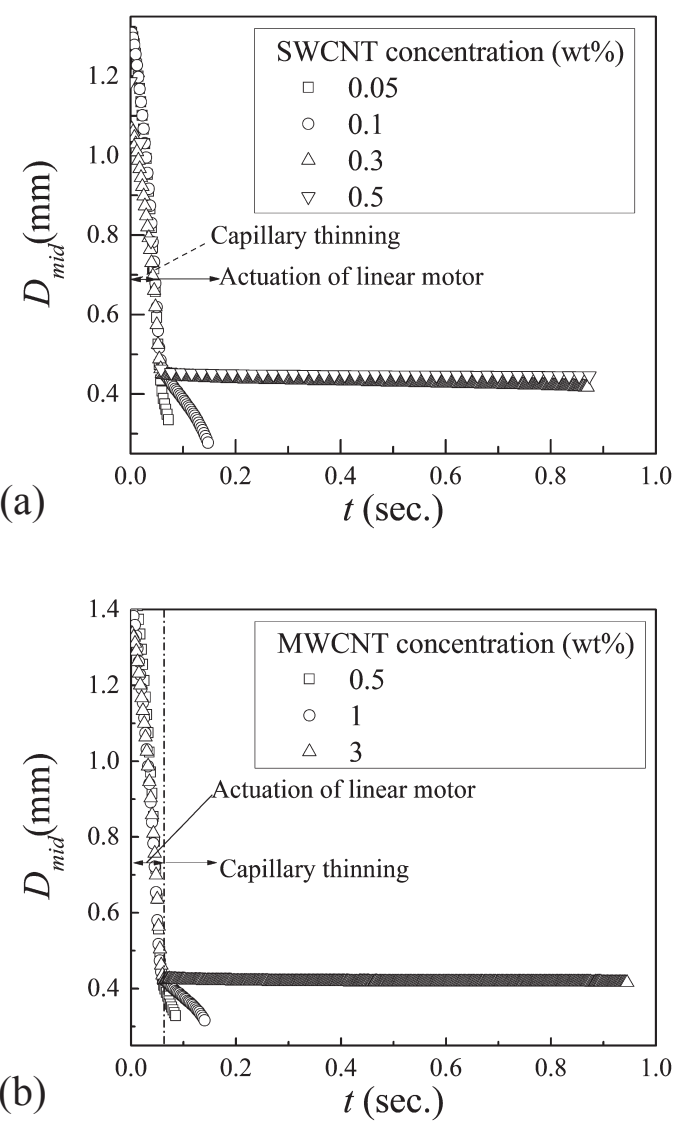

Fig. 7. Mid-diameter profiles for (a) SWCNT dispersions and (b) MWCNT dispersions. The dispersing liquid is CD9043. function of the concentration. The dispersing photopolymer CD9043 is a Newtonian fluid with a shear viscosity of $0.071 \mathrm{~Pa} \cdot \mathrm{s}$, and its extensional viscosity is therefore $0.213 \mathrm{~Pa} \cdot \mathrm{s}$. Compared with this value of $0.213 \mathrm{~Pa} \cdot \mathrm{s}$, the presence of the dispersed SWCNTs and MWCNTs increased the apparent extensional viscosity significantly. The increase due to the SWCNTs is more than two orders of magnitude higher than that due to the MWCNTs at a concentration of $0.5 \mathrm{wt} \%$. Batchelor $^{23)}$ analyzed the extensional flow of a non-dilute suspension of long elongated particles and provided the following explicit solution for macroscopic extensional viscosity:

$$
\eta_{E}=\eta_{0}\left[3+\frac{4 \phi(l / d)^{2}}{3 \ln (\pi / \phi)}\right]
$$

where $\phi$ is the volume concentration of the particles and $l / d$ is particle aspect ratio. If $l / d$ is sufficiently greater than 1 , Eq. (3) predicts the extensional viscosity to be nearly proportional to $(l / d)^{2}$. As stated in Section 2.1, the diameter and the length were typically $2 \mathrm{~nm}$ and a few microns for the SWCNTs and $15 \mathrm{~nm}$ and $3 \mu \mathrm{m}$ for the MWCNTs. Therefore, the aspect

(a)

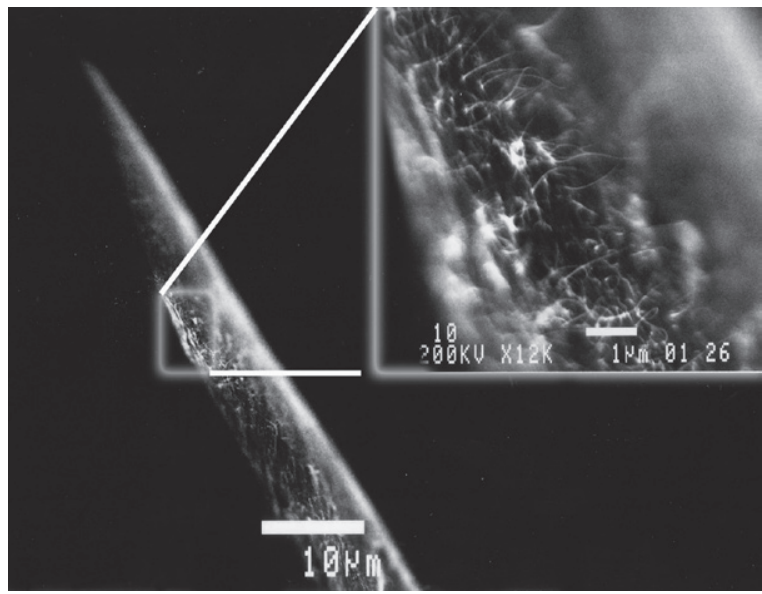

(b)

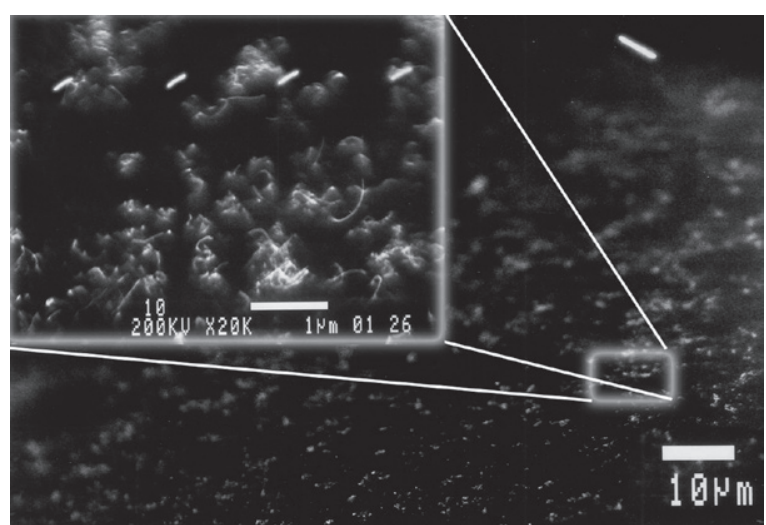

Fig. 8. Surface morphology of the solidified CNT dispersions of UAP75 for (a) $0.1 \mathrm{wt} \%$ SWCNT and (b) $0.5 \mathrm{wt} \%$ MWCNT. The dispersing photopolymer is UAP-75. 
ratio $l / d$ was nearly 1000 for the SWCNTs and about 200 for the MWCNTs. We estimated the extensional viscosity using these aspect ratios and Eq. (3), and show these in Fig. 9. By assuming the density of the CNTs to be $1750 \mathrm{~kg} / \mathrm{m}^{324}$ for the SWCNTs and $2000 \mathrm{~kg} / \mathrm{m}^{3}$ for the MWCNTs, we converted the CNT concentration from a volumetric to a weight percentage. For a SWCNT dispersion of $0.05 \mathrm{wt} \%$, Eq. (3) predicts an extensional viscosity consistent with the measured value. However, the dispersions with concentrations greater than $0.05 \mathrm{wt} \%$ had an apparent extensional viscosity that was greater than that predicted by Eq. (3), and this deviation increased with concentration. Ma et al. ${ }^{12)}$ carried out filament stretching experiments for SWCNTs that had an aspect ratio of 200. The viscosity of the dispersing liquid was $10 \mathrm{~Pa} \cdot \mathrm{s}$, which was 140 times higher than that of the CD9043 used in this study. The concentration was 0.05 to $0.3 \mathrm{wt} \%$, in the same range as the present study. They obtained an apparent extensional viscosity that was consistent with Eq. 3 for a

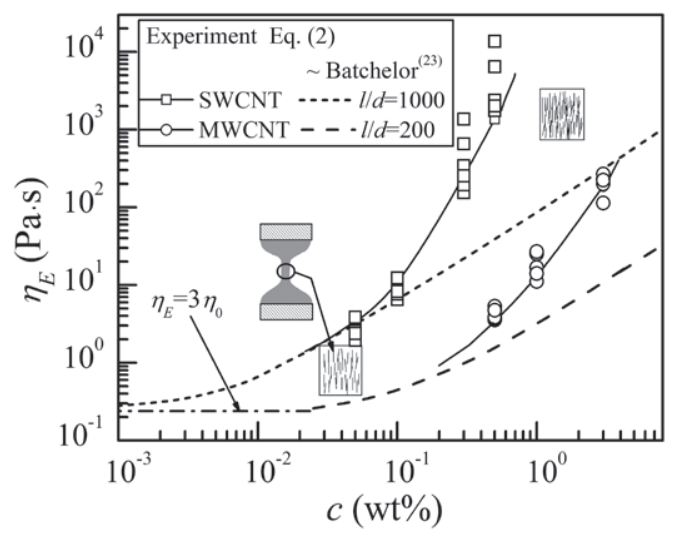

Fig. 9. Extensional viscosity for the SWCNT and MWCNT dispersions. The dispersing liquid is CD9043. The illustrations show the predicted distributions of dispersed CNTs.

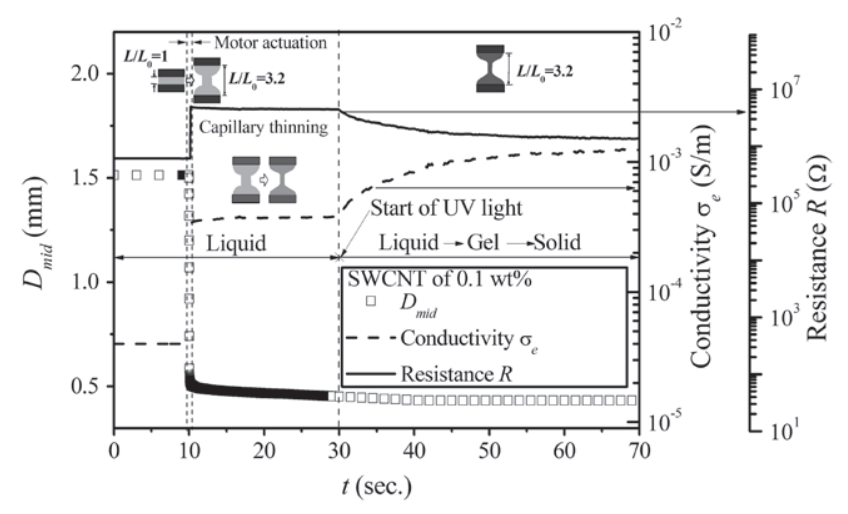

Fig. 10. Temporal variation in mid-diameter and electrical characteristics for the SWCNT dispersion of $0.1 \mathrm{wt} \%$. The dispersing liquid is CD9043.
CNT aspect ratio of 180 . Their value of $\eta_{E} / \eta_{0}$ was 2.7 - 8.2. The filament stretching experiments of Tiwari et al. ${ }^{13)}$ used MWCNTs with an aspect ratio of 200 - 500. The viscosity of the dispersing liquid was $0.79 \mathrm{~Pa} \cdot \mathrm{s}$, which was 11 times that of CD9043. These authors mainly discussed the effect of nonNewtonian behavior, based on a Herschel-Bulkley model. The value of $\eta_{E} / \eta_{0}$ for a concentration of $0.75 \mathrm{wt} \%$ was $13-1300$, the range of which was comparable with our own range of 10 $-10^{4}$ in this study.

In summary, the rheology of CNT dispersions depends on the method of dispersal, the viscosity of the dispersing liquid, the CNT aspect ratio, and the concentration. Notwithstanding the foregoing discussion, only a few studies have ever been conducted on the extensional rheology of CNT dispersions. However, the results of our own study, and those of Ma et al. ${ }^{12)}$, suggest that Eq. (3) may be used to give reasonable predictions, at least at low concentrations.

\subsection{Electrical Conductivity}

We measured the electrical resistance of the thread stretched between the disk electrodes. The measurements were conducted both for the liquid thread in the absence of irradiation by UV light and for the thread solidified by UV light. Figure 10 shows an example of the variation in the electrical properties of the solidified thread with time. Two linear motors were activated at $t=10 \mathrm{~s}$, and the velocity was increased exponentially according to Eq. (1). This stretching rapidly reduced the diameter of the thread and increased its electrical resistance between the disk electrodes. For the following $20 \mathrm{~s}$, the capillary thinning gradually reduced both the thread diameter and the electrical resistance. After the UV light was switched on at $t=30 \mathrm{~s}$, the diameter of the thread remained constant and the electrical resistance reduced. The electrical resistance is changed both by the shape of the thread and the alignment of the CNTs, the effects of which will be discussed separately. The measured electrical resistance $R$ is generally proportional to the material thickness $h$ and inversely proportional to the cross sectional area $A$. The resistivity $\rho_{e}$ was obtained using

$$
\rho_{e}=R \frac{A}{h}
$$

and is independent of the shape of the material.

In order to calculate the resistivity of the stretched liquid bridge, its shape was divided into two sections. The first section is the truncated cone on the face of each disk electrode, and the second is the central thread that connected the two truncated cones, as illustrated in Fig. 3. The image 
of the thread during stretching was captured using a CCD camera, from which we obtained the changes in the length of the central thread $h_{t}$ and the height of the truncated cones hc, assuming that the two cones are identical. The total resistance $R$ between the disk electrodes is $R=R_{\tau}+2 R_{c}$, where $R_{t}$ is the resistance $\rho_{e}\left(4 h_{t} / \pi a^{2}\right)$ of the central thread and $R_{c}$ is the resistance $\rho_{e}\left(4 h_{c} / \pi a b\right)$ of each truncated cone. The constant $a$ represents the diameter of the central thread, and $b$ is the diameter of the base of the truncated cones that abutted the electrode. The CNT dispersion in each section is subject to different stretching strain during the process in which the orientations of the CNTs are changed from their initially random state to their being aligned in the direction of stretching. The resistivity of each section is therefore different. Wang Q. et al. ${ }^{11)}$, for example, measured a higher conductivity in the direction of stretching than in the direction perpendicular to it for the case of a SWCNT composite. However, in our case the conductivity was only measured in the same direction as the stretching. In addition, it may be seen that the total resistance $R$ is approximately equal to the resistance $R_{t}$ because $R_{t} / R_{c}=b h_{t} / a h_{c}>1$. We therefore simply assumed the resistivity $\rho_{e}$ to be uniform along the entire thread. The resistivity may calculated from the measured resistance using:

$$
\rho_{e}=\frac{\pi R a^{2} b}{4\left(h_{t} b+2 h_{c} a\right)}
$$

The electrical conductivity $\sigma_{e}$ was calculated by taking the inverse of the resistivity. The example of $\sigma_{e}$ calculated in this way is shown in Fig. 10. The stretching by the linear motors increased the electrical conductivity by an order of magnitude from its initial value, and the subsequent capillary thinning maintained the conductivity approximately constant. The solidification began at $t=30 \mathrm{~s}$, after which the conductivity increased by a further order of magnitude. The increase in conductivity was therefore a result of the stretching of the thread for a short time interval and by the subsequent solidification. The thread stretching changes the orientations of CNTs from their initial random state to their being aligned in the direction of stretching. The solidification shrinks the dispersing polymer and makes the contact between CNTs tight. These changes seem to cause the increase in conductivity.

The measured conductivities are shown in Fig. 11 as a function of concentration. In order to assess the effect of the stretching, the conductivities of the threads are shown both before and after stretching. In addition, in order to determine the effect of the solidification, the conductivities were compared before and after irradiation by UV light. The conductivity of the stretched liquid was the value obtained just before irradiation. The stretching and the solidification increased the conductivity in the measured range of concentration for both the SWCNT and MWCNT dispersions. The percolation threshold was approximately $0.1 \mathrm{wt} \%$ for both the SWCNT and MWCNT dispersions. At concentrations higher than this percolation threshold, the increases in conductivity that occurred as a result of the stretching and solidification were slightly greater. Balberg et $a{ }^{25)}$ and Balberg ${ }^{26)}$ discussed the general relationship between the percolation threshold of stick-like particles and the excluded volume associated with these particles. Their result for the percolation threshold $p_{c}$ is expressed as:

$$
p_{c}=1-\exp \left(-\frac{1.4 V}{V_{e}}\right)
$$

where $V$ is the volume of the individual filler, and $V_{e}$ is the average excluded volume defined as

$$
V_{e}=\frac{4 \pi}{3} d^{3}+2 \pi d^{2} l+\frac{\pi}{2} d l^{2}
$$

where $d$ is diameter and $l$ is the length of the sticks. The average on $V_{e}$ is taken over the randomly oriented distribution of the sticks. Celzard et al. ${ }^{27)}$ applied Eq. (6) to the percolation threshold for polymer-carbon-fiber composites and obtained reasonably good agreement between calculated and experimental values of the percolation threshold. Equation (6) gives a percolation threshold $p_{c}=0.00070$ for SWCNTs and $p_{c}=0.0017$ for MWCNTs. These volume fractions may be converted to weight concentrations $p_{c}=0.12 \mathrm{wt} \%$ for SWCNTs and $p_{c}=0.35 \mathrm{wt} \%$ for MWCNTs. These percolation thresholds

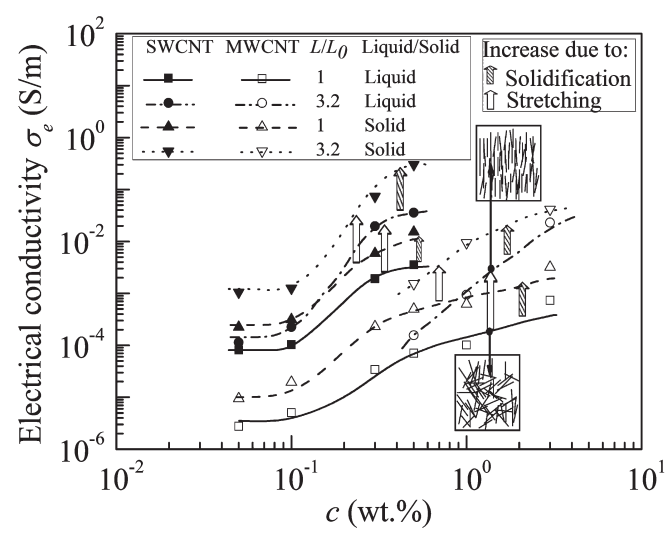

Fig.11. Electrical conductivity of the CNT dispersions as a function of concentration. The dispersing liquid is CD9043. The illustrations show the change in the predicted CNT orientation from isotropy to non-isotropy. 
are similar to the threshold of $0.1 \mathrm{wt} \%$ observed here.

It may be seen that this experimentally determined threshold is roughly the same as the concentration at which the apparent extensional viscosity began to deviate from the theoretical prediction of Batchelor, as shown in Fig. 9. This agreement points to a close relationship between the increases in apparent extensional viscosity and electrical conductivity. The deviations shown in Fig. 9 suggest the incidence of the nonaligned and entangled network structure of CNTs, which may induce the percolative increase in conductivity. As mentioned above, the increase in conductivity that takes place due to stretching is slightly higher at concentrations above the percolation threshold. The electric percolation is influenced by the CNT alignment. Although the electrical percolation of CNT composites has been studied by many researchers as reviewed by Bauhofer and Kovacs ${ }^{4}$, there have been few studies of the alignment effect. Du et al. ${ }^{28)}$ investigated the alignment effect of conductive sticks using Monte Carlo simulations and experiments, and showed that the highest conductivity occurred for slightly aligned sticks, rather than for isotropic or completely aligned sticks. The CNT alignment in the dispersion is initially isotropic between the electrodes. The slightly aligned dispersion of the CNTs may be induced by the thread stretching above the percolation threshold due to the incidence of their network structure.

\section{CONCLUSIONS}

The relative viscosity was measured under steady shear for both SWCNT and MWCNT dispersions, and the influence of the viscosity of the dispersing liquid was investigated. At low shear rates, a dispersing liquid with a low viscosity increased the relative viscosity more notably than a dispersing liquid with a high viscosity, in the case of SWCNT dispersions. The shear viscosity approaches the same value regardless of the viscosity of the dispersing liquid. This independence suggests that at low shear rates the shear viscosity was largely determined by the microstructure of the SWCNTs rather than the viscosity of dispersing liquid.

During capillary thinning, the apparent extensional viscosity approached the theoretical values predicted by Batchelor for SWCNT and MWCNT dispersions at low concentrations. However, the apparent extensional viscosity increased significantly from the theoretically predicted values at high concentrations. During capillary thinning, we measured the electrical conductivity of a single thread of the dispersion. The thread stretching and solidification of the photopolymer increased the electrical conductivity in both cases. The concentration at the electrical percolation threshold was approximately the same as the concentration at which the apparent extensional viscosity deviated from the theoretical prediction of Batchelor. This result suggests that the increase in apparent extensional viscosity was brought about by the percolated network structure of the CNTs.

\section{REFERENCES}

1) Iijima $S$, Nature, 354, 56 (1991).

2) Andrews R, Weisenberger MC, Curr Opin Solid State Mater Sci, 8, 31 (2004).

3) Harris PJF, Int Mater Rev, 49(1), 31 (2004).

4) Bauhofer W, Kovacs JZ, Compos Sci Technol, 69, 1486 (2009).

5) Sahoo NG, Rana S, Cho JW, Li L, Chan SH, Prog Polym Sci, 35, 837 (2010).

6) Ma P-C, Siddiqui NA, Maro G, Kim J-K, Composites Part A, 41, 1345 (2010)

7) Fan S, Science, 283, 512 (1999).

8) Sohn J, Lee S, Song YH, Choi SY, Cho KI, Nam KS, Current App Phys, 1, 61(2001).

9) Vink TJ, Gillies M, Kriege JC, Van de Laar H W J J. Appl Phys Lett, 83, 3552 (2003).

10) Thostenson ET, Chou TW, J phys D: App phys, 35, 77 (2001).

11) Wang Q, Dai J, Li W, Wei Z, Jiang J, Comp Sci Tech, 68, 1644 (2006).

12) Ma A WK, Chinesta F, Tuladhar T, Mackley MR, Rheol Acta, 47, 447 (2008).

13) Tiwari M K, Bazilevsky A V, Yarin A L, Megaridis C M, Rheol Acta, 48, 597 (2009).

14) McKinley GH, Rheol Rev, 2005, 1 (2005).

15) Darsono N, Mizunuma H, Obara H, Appl Rheol, 21, 63566 (2011).

16) Mckinley GH, Tripathi A, J Rheol, 44, 653 (2000).

17) Papageorgiou DT, Phys Fluid, 7(7), 1529, (1995).

18) Anna SL, McKinley GH, J Rheol, 45, 115 (2001).

19) Vadillo DC, Tuladhar TR, Mulji AC, Jung S, Hoath SD, Mackley MR, J Rheol, 54, 261(2010).

20) Tuladhar T, Mackley M, J Non Newtonian Fluid Mech, 148(1-3), 97 (2008).

21) Rodd LE, Scott TP, Cooper-White JJ, Appl Rheol, 15(1), 12, (2005).

22) Kinloch IA, Roberts SA, Windle AH, Polym, 43, 7483, (2002).

23) Bacthelor GK, J Fluid Mech, 46, 813, (1971).

24) Shaffer MSP, Windle AH, Macromolecules, 32, 6864 (1999).

25) Balberg I, Anderson $\mathrm{CH}$, Alexander S, Wagner N, Phys Rev B, 30, 3933 (1984).

26) Balberg I, Phys Rev B, 33, 3618 (1986).

27) Celzard A, McRae E, Deleuze C, Dufort M, Furdin G, Marêché J, Phys Rev B, 53, 6209 (1996).

28) Du F, Fischer J, Winey K, Phys Rev B, 72, 1 (2005). 